\title{
CONVOLUTIONS AND HÖLDER-TYPE INEQUALITIES FOR A CERTAIN CLASS OF ANALYTIC FUNCTIONS
}

\author{
JUNICHI NishiwaKI, SHIGEYOSHI OWA AND H. M. SRIVASTAVA
}

Abstract. Applying the coefficient inequalities for functions $f(z)$ belonging to a certain class $\mathscr{M} \mathscr{D}(\alpha, \beta)$ of normalized analytic functions in the open unit disk $\mathbb{U}$, two subclasses $\mathscr{M}_{1}(\alpha, \beta)$ and $\mathscr{M}_{2}(\alpha, \beta)$ are defined. The object of the present paper is to derive some properties for functions $f(z)$ in the classes $\mathscr{M}_{1}(\alpha, \beta)$ and $\mathscr{M}_{2}(\alpha, \beta)$ involving their generalized convolution by utilizing methods based upon the Hölder-type inequalities.

Mathematics subject classification (2000): 30C45, 30A10, 26 D07.

Key words and phrases: Analytic functions, uniformly starlike functions, Hölder inequality, Hadamard product (or convolution), elliptic, parabolic and hyperbolic domains.

\section{REFERENCES}

[1] J. H. CHOI, Y. C. KIM AND S. OwA, Generalizations of Hadamard products of functions with negative coefficients, J. Math. Anal. Appl. 199 (1996), 495-501.

[2] S. KANAS AND H. M. SRIVASTAVA, Linear operators associated with $k$-uniformly convex functions, Integral Transform. Spec. Funct. 9 (2000), 121-132.

[3] J. NISHIWAKI AND S. OWA, Certain classes of analytic functions concerned with uniformly starlike and convex functions, Appl. Math. Comput. 187 (2007), 425-432.

[4] J. NishIWAKI AND S. OWA, An application of Hölder inequality for convolution, Proc. Japan Acad. Ser. A Math. Sci. (submitted).

[5] J. NiSHIWAKI AND S. OWA, Some properties of certain analytic functions, Sūrikaisekikenkyūsho Kokyūroku, 1538 (2007), 4-50; see also Acta Univ. Apulensis Math. Inform. 13 (2007), 109-116.

[6] S. OWA AND H. M. SRIVASTAVA, Some generalized convolution properties associated with certain subclasses of analytic functions, J. Inequal. Pure Appl. Math. 3 (3) (2002), Article 42, 1-13. 